\title{
FLEXIBLE BOOMS, MOMENTUM WHEELS, AND SUBTLE GRAVITY-GRADIENT INSTABILITIES
}

\author{
J. W. Hunt and J. C. Ray \\ The Johns Hopkins University Applied Physics Laboratory \\ Laurel, Maryland 20723
}

\begin{abstract}
A gravity-gradient boom and a momentum wheel provides a passive, three-axis attitude control system for a small satellite requlring $10^{\circ}$ Earth-oriented pointing in a low Earth orbit. The Polar BEAR satellite is a small satellite using just such a system that has experienced unexpected attitude instabillties during some of its full-sun orbit periods. This paper examines the attitude dynamics and disturbances associated with gravity-gradient/momentum-wheel systems in an attempt to identify potential destabilizing mechanisms common to the configuration. Polar BEAR is not the only such configuration to experience problems in full sun, and several other examples are briefly discussed. Although we place particular emphasis on trying to understand Polar BEAR's anomaly, its performance may be symptomatic of problems with the flexible-boom/momentum-wheel conflguration.
\end{abstract}

\section{INTRODUCTION}

Many satellites take advantage of the inverse-square gravitational force to provide passive, Earth-oriented attitude control. The gravity-gradient torque on an asymmetric satellite forces the principal axis of minimum moment-ofinertia to be aligned with the local vertical ${ }^{1}$. Many gravitygradient stabilized satellites use extendable booms to achieve a favorable moment-of-inertia distribution providing 2 -axis (pitch and roll) attitude control. The addition of a constantspeed momentum wheel to a gravity-gradient stabilized satellite provides gyroscopic stiffness to passively stabilize the third (yaw) axis ${ }^{2}$.

The Polar BEAR satellite ${ }^{3}$ (Figure 1), launched in November, 1986, into a nearly circular polar orbit of 1000 $\mathbf{k m}$ altitude, was designed to provide three-axis stabilization to within $\pm 10^{\circ}$ for its Earth-pointing payload. Its attitude control system uses an 18.3-m interlocked BI-STEM gravitygradient boom, a constant-speed momentum wheel, and a boom-mounted magnetically-anchored eddy-current damper to provide passive, three-axis stabilization. Until midFebruary of 1987 the Polar BEAR mission proceeded as designed and its attitude performance was nominal.

As Polar BEAR entered its first period of fully sunlit orbits in mid-February of 1987 , its attitude performance degraded significantly. Within a 5 day period Polar BEAR's peak yaw angle increased more than $50^{\circ}$, the peak pitch angle increased more than $30^{\circ}$, and the peak roll angle increased more than $10^{\circ}$. Polar BEAR's anomalous attitude motion continued until the satellite finally inverted in May,

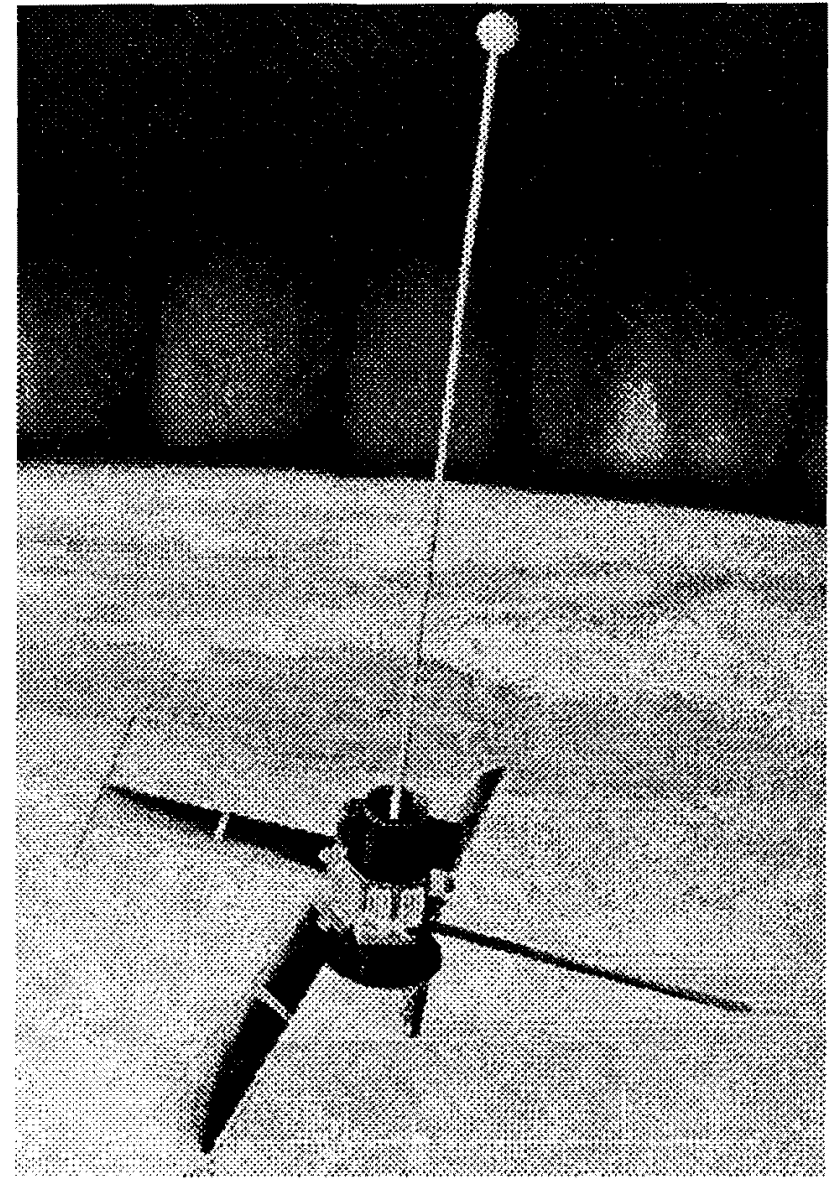

Figure 1 The Polar BEAR spacecraft.

$1987^{4}$. After reentering eclipsed orbits, Polar BEAR was 
reinverted and again performed nominally. During its second period of fully sunlit orbits in the fall of 1987, Polar BEAR's attitude performance was very good'; however, Polar BEAR again experienced large attitude excursions during both full-sun periods of 1988 .

Polar BEAR's anomalous attitude motion happened despite attempts in the design process to minimize such occurrences. Polar BEAR was a follow-up to the HILAT satellite launched in June, $1983^{\circ}$. HILAT's attitude control system is similar to Polar BEAR's, however, the gravitygradient boom is a torsionally-weak, overlapped tape boom, and there is no eddy-current damper. HILAT generally met its pitch and roll control requirements of $\pm 10^{\circ}$ but its yaw performance exceeded the attitude specification about $20 \%$ of the time? HILAT's yaw motion increased as the sun approached the normal to the orbit plane (full-sun orbits) but the yaw angle stayed below $40^{\circ}$.

The HILAT attitude excursions are believed to be caused by thermal bending of its gravity-gradient boom. Thermally induced vibrations of torsionally-weak booms, frequently

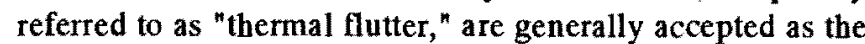
cause of anomalous behavior on other gravity-gradient stabilized spacecraft ${ }^{89}$. In the case of HILAT, the gyroscopic stiffness provided by the momentum wheel was apparently insufficient to prevent the instabilities associated with thermal flutter. Recommended solutions to the thermal flutter problem are to increase the torsional rigidity of the gravity-gradient boom and increase system damping. Polar BEAR's boom was a torsionally-stiffer zippered boom and it had an eddy current damper; why, then, the anomalous attitude motion and inversions? The Naval Research Laboratory (NRL) has experienced similar anomalous motion with gravity-gradient stabilized spacecraft using momentum wheels, eddy current dampers, and zippered booms ${ }^{10}$ so the problem is not specific to Polar BEAR.

While gravity-gradient/wheel satellites with flexible booms have experienced unexpected attitude excursions, some satellites with much stiffer booms have exhibited unexpected attitude performance. The GEOSAT satellite has a very stiff scissors boom, a momentum wheel, and an eddycurrent damper ${ }^{11}$. Although GEOSAT's mission was extremely successful, its attitude performance was slightly degraded at certain times of the year. These periods of slightly degraded performance were strongly correlated with the position of the sun relative to the orbit plane ${ }^{12}$. While not adversely affecting the GEOSAT mission, these unexpected periods of slightly degraded performance are another example of the uncerta inties that accompany gravitygradient stabilized systems.
The NOVA satellite ${ }^{13}$ is also of this configuration, and includes a relatively stiff boom called an Astromast. Like GEOSAT, NOVA has been quite successful, but experienced degraded gravity gradient performance at certa in times of the year. There were three NOVA satellites; data from NOVA II (launched in 1988) provides an example of this phenomenon. Figure 2 is a trend plot of NOVA II yaw

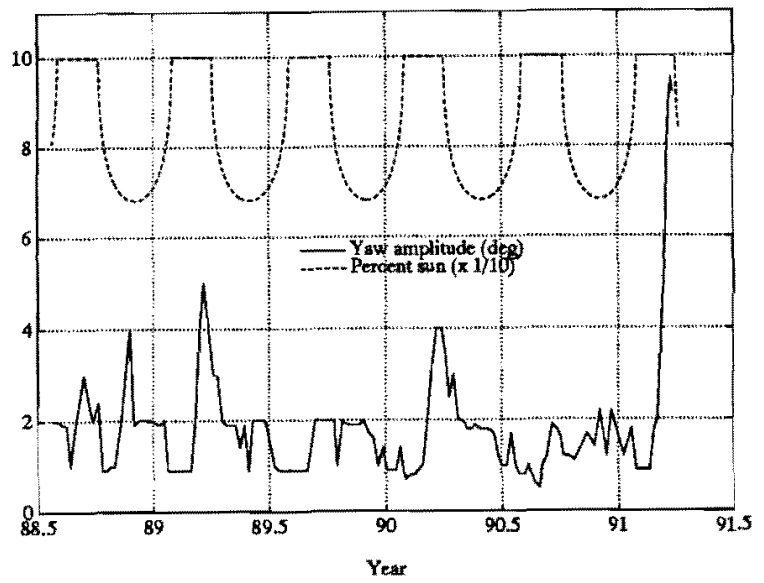

Figure 2 Correlation of NOVA's yaw amplitude with orbital sunlight.

amplitude and percent sun from launch through early 1991 . In its polar orbit NOVA bas two full sun periods each year; the yaw librations are noticeably greater toward the end of the first full sun period each year, but not the second. The 1991 anomaly is the worst yet; this is interesting in that the solar activity (and hence atmospheric drag) was near maximum at this time. The possible connection of atmospheric disturbance with gravity gradient performance is discussed further below.

In this paper we discuss passive, momentum-wheel augmented, gravity-gradient stabilized satellites and some of the problems experienced with their configuration. We begin by presenting an overview of the attitude dynamics of this class of satellites and then discuss some of the disturbances they may encounter. Finally, we look in more detail at the boom flexure of Polar BEAR and present simulation results that show instabilities similar to those observed on Polar BEAR.

\section{GRAVITY-GRADIENT/MOMENTUM WHEEL ATTITUDE DYNAMICS}

Before proceeding further it is appropriate to define what we mean by satellite attitude. Satellite attitude refers to the rotational orientation of the satellite's body axes relative to some reference triad of Cartesian axes. For a

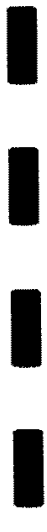

I

।

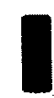

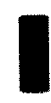

】

I

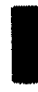

| 


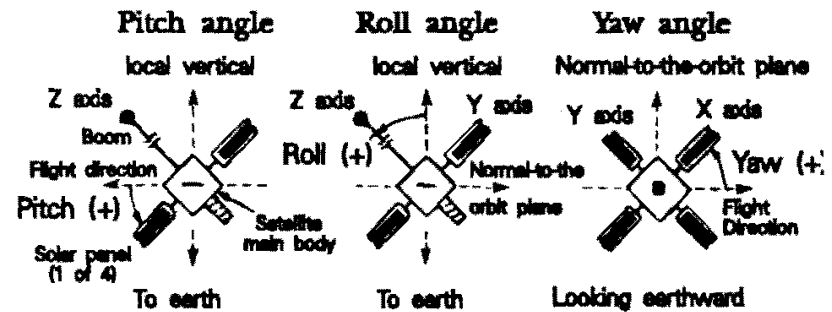

Figure 3 spacecraft attitude angles.

gravity-gradient-stabilized spacecraft, the reference system is called the local vertical system, which consists of the outbound local vertical, the normal-to-the-orbit plane, and the vector that completes the right-hand set (along the velocity vector for a circular orbit).The orientation (or attitude) of the satellite relative to the local vertical system is defined by a three Euler rotation angles, where pitch is "nose" down (positive) or up (negative), roll is "left wing" up (positive) or down (negative), and yaw is nose left (positive) or right (negative). Each attitude angle is shown in terms of spacecraft motion in Figure 3. The system of body axes $(x, y, z)$ we use are defined such that for zero attitude errors the body $y$-axis is parallel to the orbit normal, the $z$ axis is parallel to the outbound local vertical, and the $x$-axis is along the velocity vector for a circular orbit.

As mentioned previously, the gravity-gradient torque acting on an asymmetric satellite tends to align the principal axis of minimum moment-of-inertia with the local vertical. The gravity-gradient torque, $T_{g 8}$, results from the inverse square gravitational law expressed as:

$$
T_{g g}=3 \frac{\mu}{r^{3}}[\hat{r} \times(I \cdot \hat{r})]
$$

where $\mu$ is the Earth's gravitational constant, $I$ is the satellite's inertia tensor, and $r$ and $\hat{\boldsymbol{r}}$ are the distance and the unit vector from the Earth's center to the center of mass of the satellite. For a nearly circular orbit, the quantity $\mu / r^{3}$ is frequently replaced by the square of the average orbital rate, $\omega_{0}{ }^{2}$. Note from the equation that the torque is normal to the local vertical $(\hat{r})$ and is inversely proportional to the cube of the geocentric distance. Gravity-gradient stabilization is most effective for low Earth orbits; however, the orbit must be high enough that the gravity-gradient torques can overcome aerodynamic disturbance torques.

The satellites discussed in this paper all have a common ittitude configuration consisting of a gravity-gradient boom Ind a constant-speed momentum wheel aligned along the ody $y$-axis (pitch axis). Combining the gravity-gradient orque expression with Euler's rigid body dynamics quations modified to account for the momentum whe $\mathrm{l}^{14}$, and linearizing about the equilibrium state of zero roll, pitch, and yaw angles yields the following equations of motion:

$$
\begin{gathered}
I_{y} \theta_{p}+3 \omega_{o}^{2}\left(I_{x}-I_{z}\right) \theta_{p}-T_{y} \\
I_{x} \theta_{r}+\left[4 \omega_{o}^{2}\left(I_{y}-I_{z}\right)+\omega_{o} h_{w}\right] \theta_{r} \\
-\left[\left(I_{y}-I_{x}-I_{z}\right) \omega_{o}+h_{w}\right] \theta_{y}-T_{x} \\
I_{z} \theta_{y}+\left[\left(I_{y}-I_{x}\right) \omega_{o}^{2}+\omega_{o} h_{w}\right] \theta_{y} \\
+\left[\left(I_{y}-I_{x}-I_{z}\right) \omega_{o}+h_{w}\right] \theta_{r}=T_{z}
\end{gathered}
$$

where $\theta_{p}, \theta_{p}$ and $\theta_{y}$ are the pitch, roll, and yaw errors with a single dot indicating the first derivative with respect to time (angular rate), and the double dot indicating the second derivative (angular acceleration). The body moments-ofinertia are indicated by $I_{x}, I_{y}$ and $I_{x}$ and the constant wheel angular momentum about the spacecraft y-axis is $h_{w} T_{x}, T_{p}$ and $T_{z}$ are the torque components on the body for all torques exclusive of the gravity-gradient torque.

Equations (2) above show that for small angle motion, pitch is decoupled from roll and yaw. Roll and yaw, however, are coupled to one another through the bias wheel momentum, $h_{w}$ and the orbital rate term, $\left(I_{y}-I_{x}-I_{z}\right) \omega_{0}$. In pitch, the gravity-gradient restoring torque is proportional to the pitch error with libration frequency $\omega_{g}=\left[3\left(I_{x}-I_{z}\right) / I_{y}\right]^{h} \omega_{0}$. Pitch stability requires that $I_{x}>I_{z}$. For Polar BEAR, $I_{x}=$ $934 \mathrm{~kg}-\mathrm{m}^{2}, I_{y}=937 \mathrm{~kg}-\mathrm{m}^{2}, I_{z}=29 \mathrm{~kg}-\mathrm{m}^{2}$, and $\omega_{0}$ is approximately $0.001 \mathrm{rad} / \mathrm{s}$ which gives a pitch libration period of 62 minutes.

For $\Delta I=I_{x}-I_{z}$ and $I_{x} \approx I_{y}$, the coupled roll/yaw equations have characteristic equation

$$
\begin{gathered}
\left(s^{2}+\frac{\omega_{o} h_{w}+4 \omega_{0}^{2} \Delta I}{I_{x}}\right)\left(s^{2}+\frac{\omega_{o} h_{w}}{I_{z}}\right) \\
+s^{2}\left(\frac{h_{w}}{I_{x}}-\frac{I_{z}}{I_{x}} \omega_{o}\right)\left(\frac{h_{w}}{I_{z}}-\omega_{o}\right)=0
\end{gathered}
$$

which has roots $\left(\omega_{1}, \omega_{2}\right)$, the two natural frequencies associated with roll and yaw motion. The high frequency roll/yaw mode (6.1-min period for Polar BEAR) can be thought of as the motion of the satellite's wheel axis about the system's total angular momentum vector in a cone (nutation), while the low frequency roll/yaw motion (78-min period) corresponds to a slow precession of the total angular momentum vector resulting from gravity-gradient torques. The Polar BEAR anomaly appeared as a build-up in the amplitude of the high frequency roll/yaw mode which coupled into pitch as the angles increased.

As noted by Pisacane ${ }^{15}$, it is known (see Garber ${ }^{16}$ ) that a constant pitch torque de-stabilizes the roll/yaw motion of 
a gravity-gradient stabilized satellite. However, Garber's analysis does not apply directly to a case such as Polar Bear with a momentum wheel. In the presence of a small constant pitch torque $M_{b}$, the pitch motion will consist of gravity-gradient libration about a non-zero bias angle $\theta_{b}$, given by:

$$
\theta_{b}-\frac{M_{b}}{3 \omega_{o}^{2} \Delta l}
$$

With $I_{x} \approx I_{y}$, the equations of motion linearized about this pitch bias angle become:

$$
\begin{gathered}
I_{y} \theta_{p}+\left(3 \omega_{o}^{2} \Delta I \cos 2 \theta_{b}\right) \theta_{p}-T_{y} \\
I_{x} \theta_{r}+\left[\omega_{o}\left(h_{w}+\omega_{o} \Delta I\right)+3 \omega_{e}^{2} \Delta I C_{b}^{2}\right] \theta_{r} \\
-\left(h_{w}-I_{z} \omega_{a}\right) \theta_{y}+\left(3 \omega_{o}^{2} \Delta I S_{b} C_{b}\right) \theta_{y}-T_{x} \\
I_{z} \ddot{\theta}_{y}+\omega_{o} h_{w} \theta_{y}+\left(h_{w}-I_{z} \omega_{o}\right) \theta_{p}-T_{z}
\end{gathered}
$$

where $\left(S_{b}, C_{b}\right)=\left(\sin \theta_{b}, \cos \theta_{b}\right)$. Note that $\theta_{p}$ is now with respect to the biased equilibrium, and that $\theta_{b}$ does not enter the yaw equation $(5 \mathrm{~b})$. The pitch motion remains uncoupled from roll/yaw, but the libration frequency is reduced (by the factor $\left.\left[\cos \left(2 \theta_{b}\right)\right]^{k}\right)$ from that with no pitch bias.

Stability of the roll/yaw motion may be analyzed by taking the Laplace transform of $(5 b)$ and $(5 c)$ with $T_{x}=T_{z}=$ 0 , giving:

$$
\begin{aligned}
& {\left[\begin{array}{c}
I_{x} s^{2}+\omega_{0}\left(h_{w}+\omega_{0} \Delta I\right)+3 \omega_{0}^{2} \Delta I C_{b}^{2} \\
\left(h_{w}-I_{z} \omega_{o}\right) s
\end{array}\right.} \\
& \left.\begin{array}{c}
-\left(h_{w}-I_{z} \omega_{o}\right) s+3 \omega_{o}{ }^{2} \Delta S_{b} C_{b} \\
s^{2} I_{z}+\omega_{o} h_{w}
\end{array}\right]\left[\begin{array}{l}
\theta_{y} \\
\theta_{y}
\end{array}\right]=0 .
\end{aligned}
$$

For small bias angles (i.e,, small bias torques), the characteristic equation of $(6)$ is:

$$
\left(s^{2}+\omega_{1}^{2}\right)\left(s^{2}+\omega_{2}^{2}\right)-\omega_{g}^{2} \theta_{b}\left(\frac{h_{w}}{I_{z}}-\omega_{o}\right) s=0,
$$

where $\left(\omega_{1}, \omega_{2}\right)$ are the roll/yaw natural frequencies at $\theta_{b}=$ 0 . Figure 4 is a root-locus plot of equation (7), showing the system characteristic root locations for all values of $\theta_{b}$. (The lower half of the symmetric s-plane is not shown.) The roll/yaw dynamics (with no damping) are stable only if all four roots are in the left-half plane. As seen in Figure 4, there is always one pair of roots in the right-half plane for any non-zero $\theta_{b}$, hence a pitch bias is always de-stabilizing. The high frequency roll/yaw mode diverges for negative (nose-up) $\theta_{b}$ while the low-frequency mode diverges for positive $\theta_{b}$. It should be emphasized that, in practice, damping is present. The effect of this de-stabilization is

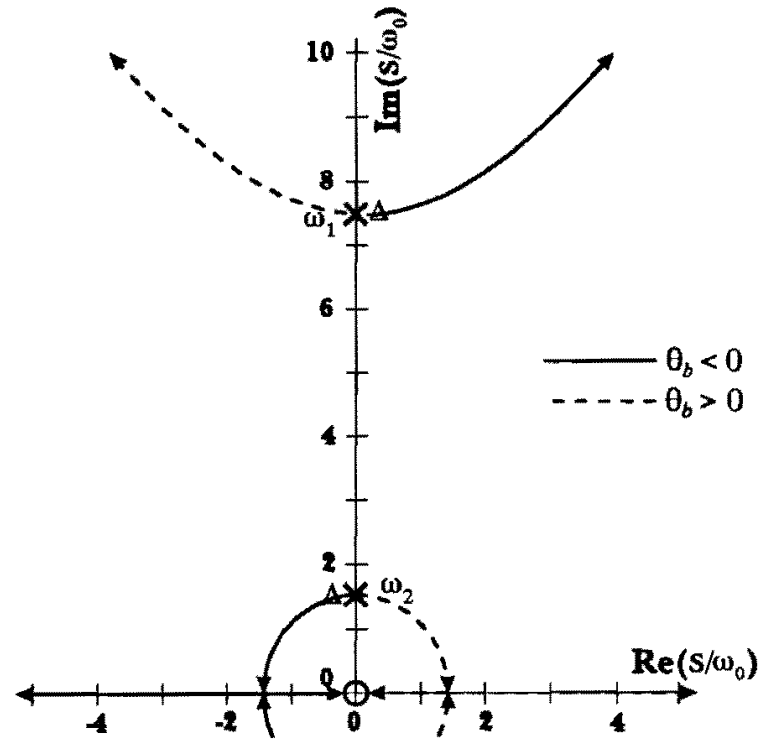

Figure 4 Root-locus for a pitch bias.

normally to lengthen the damping time constant, rather than actually cause attitude divergence.

For very small bias torques, $\theta_{b}$ will be small, and the characteristic roots will be close to the poles in Figure 4 (e.g., at the locations shown as $\Delta$ ). The root near j $\omega_{1}$ is at $\mathbf{s} \sigma+j \omega_{1}$, where $\sigma \ll \omega_{1}$. Substituting this value for $\mathbf{s}$ in (4) and retaining only first-order terms in $\sigma / \omega_{1}$ and $\sigma / \omega_{2}$ yields:

$$
\omega_{g}^{2} \theta_{b}\left(\frac{h_{w}}{I_{z}}-\omega_{o}\right)=2 \sigma\left(\omega_{2}^{2}-\omega_{1}^{2}\right)
$$

or for $\sigma=1 / \tau$ :

$$
\tau \approx \frac{2\left(\omega_{2}{ }^{2}-\omega_{1}{ }^{2}\right)}{\omega_{z}{ }^{2}\left(\frac{h_{w}}{I_{z}}-\omega_{0}\right) \theta_{b}}
$$

Similarly, the root near $\mathrm{j \omega}_{2}$ is easily shown to be approximately $-\mathrm{o}+\mathrm{j} \omega_{2}$, i.e., the real parts of both fast and slow roots have the same magnitude. Thus (9) gives the divergent time constant for positive or negative (small) pitch bias torques.

\section{ATTITUDE DISTURBANCES}

There are a number of potential attitude disturbance sources for gravity-gradient stabilized satellites. The dominant sources of attitude torques are generally the Earth's magnetic field, solar radiation pressure, and 
aerodynamic drag. Satellite flexibility, while not an external disturbance, can certainly affect the attitude of the payload. In order for the satellite to be stable, the gravity-gradient restoring torque must be able to overcome the disturbances. For Polar BEAR and its $1000 \mathrm{~km}$ orbit, the gravity-gradient restoring torque is approximately $4.7 \times 10^{-5} \mathrm{Nm} / \mathrm{deg}$.

\section{External Disturbances}

Solar radiation pressure impinging on a satellite's surfaces produces forces which result in torques about the vehicle's center of mass. Because the solar radiation at the Earth is approximately constant, the solar radiation torque depends primarily on the satellite's geometry and surface properties, and the orientation of its orbit with respect to the sun line. A satellite's geometry and surface properties are generally fixed once that satellite is deployed so variations in the solar torques result from the satellite's attitude and orbital motion relative to the sun. When the sun is in or very near the orbital plane, the solar torque is a pitch torque varying at the orbital frequency as the satellite progresses through its orbit. With the sun nearly normal to the orbit plane, the solar radiation torque tends to become an approximately constant, roll-bias torque. The magnitude of the solar pressure torque is about $1.5 \times 10^{-5} \mathrm{Nm}$ for Polar BEAR which should produce less than a degree of bias in roll.

Polar BEAR's solar panels are arranged in a windmill arrangement around the satellite. Due to this arrangement, when the sun is normal to the orbit plane the solar radiation produces a $y$-axis bias torque and a pitch bias angle. The direction of this bias differs with the side of the orbit on which the sun is located. As mentioned in the preceding section, a pitch bias destabilizes one of the roll/yaw modes while stabilizing the other. Consequently, one of the first potential causes of the Polar BEAR anomaly to be investigated was the sun/spacecraft geometry. What we found in studying the first inversion was that the pitch bias at the time should have destabilized the slow roll/yaw mode. Instead, it appeared to be the fast roll/yaw that became unstable. As Polar BEAR experienced instabilities and good performance with the sun on either side of the orbit plane, solar-induced pitch bias was necessarily ruled out as the primary cause of the Polar BEAR inversions.

In low Earth orbits, the Earth's atmosphere also interacts with the satellite surfaces to create disturbance torques on the vehicle. The aerodynamic torque is a function of atmospheric density which depends on solar activity but decays approximately exponentially with altitude. Since most of the atmosphere's interaction with the spacecraft occurs in the orbital plane, the aerodynamic torque is primarily a pitch torque. The aerodynamic torque varies around the orbit and during the day due to the density variations in the atmosphere. At Polar BEAR's $1000 \mathrm{~km}$ altitude, however, the aerodynamic torque is approximately two orders of magnitude less than the solar radiation torque. Non-linear simulations of Polar BEAR's attitude motion that included detailed aerodynamic modeling indicated no instabilities from the acrodynamic torques.

Atmospheric drag produces disturbance torques which are rich in harmonics of the orbital frequency, as well as bias torques. Although these torques are of very small amplitude at altitudes around $1000 \mathrm{~km}$, they may excite the lightly damped libration modes if the frequencies are nearly resonant. $A$ trend analysis was conducted on data from a spacecraft of configuration very similar to Polar Bear. Pitch, roll, and yaw data, sampled approximately every 100 seconds, was available for one 24 hour span per week, from December 1987 to May 1989. Each 24 hour span was analyzed in the frequency domain, from which a root-meansquare (RMS) value for three frequency bands was obtained.

Figure 5 shows the results of this trend analysis. The frequency bands represent low $(f<0.02)$, high $(f>0.03)$, and all frequencies, with $f$ in cycles per minute (cpm). Only pitch and yaw are shown; roll \& yaw are coupled and the
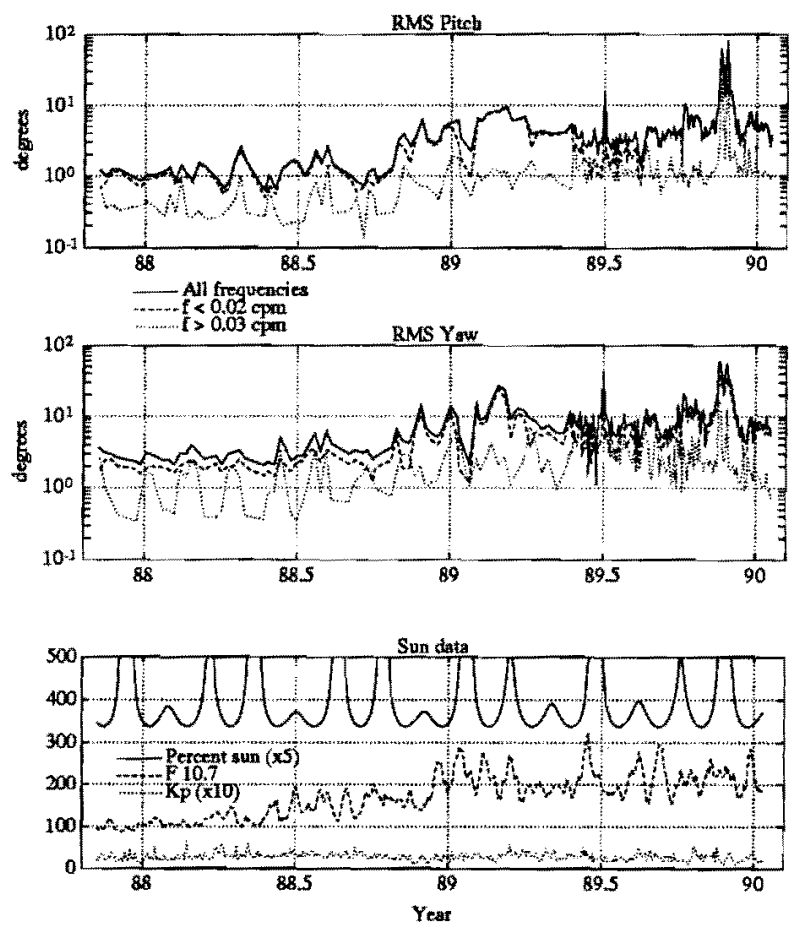

Figure 5 Attitude trends for a gravitygradient/momentum wheel stabilized satellite. 
trends are identical except for amplitude. The figure also shows the percentage of each orbit that the spacecraft is in sunlight, and two parameters which are measures of atmospheric activity: $F_{10.7}$, the smoothed $10.7 \mathrm{~cm}$ solar flux, and the magnetic index $\mathrm{K}_{\mathrm{p}}, \mathrm{F}_{10.7}$ is a direct measure of the solar activity which has a well-known 11 year cycle. The correlation with percent sun is evident. Not every full sun period results in grossly disturbed attitude, but the performance is worse in full sun on average. Note that the high frequency yaw performance is apparently better in full sun than eclipse when the overall libration amplitudes are small. This may be due to the thermal flutter effect, discussed below. There clearly is also a long-term correlation between $F_{10.7}$ and RMS attitude, both in pitch and roll/yaw. There does not appear to be any correlation with the magnetic index $\mathrm{K}_{\mathrm{p}}$. It is tempting to conclude from this that solar-induced atmospheric activity exacerbates the full sun destabilization phenomenon, whatever its cause. A physical mechanism for this connection remains unidentified.

Torques produced by the interaction of spacecraft magnetic dipoles and the local magnetic field have also been considered as a destabilizing mechanism. The total spacecraft magnetic dipole is, in general, the sum of residual dipoles in spacecraft elements, dipoles induced in spacecraft elements by the local magnetic field, and deliberate dipoles created for control purposes. Polar BEAR uses a magnetically-anchored eddy-current damper to damp attitude rates. Magnetic hysteresis rods provided rate damping following launch vehicle separation and yo-yo despin but are less effective in the gravity-gradient mode. The hysteresis rods do produce a small disturbance torque in the operational mode. Residual dipoles were trimmed to small levels in prelaunch magnetics tests. All of these magnetic effects have been modeled in simulations but none of them appear to produce instabilities as observed on Polar BEAR.

Yet another hypothesized source for a destabilizing external torque mechanism is accumulation of spacecraft charge. If the spacecraft somehow acquires a charge distribution along its long (vertical) dimension, this can be modeled as a dipole moving through the Earth's magnetic field. This will produce forces and moments on the spacecraft, which will in general consist of biases and orbital frequency harmonics. It is thus possible that either through a resonance effect or bias torques this could destabilize the gravity gradient equilibrium. Although we have not succeeded in developing a plausible physical mechanism for accumulation of the hypothesized charge, it would probably be very sensitive to spacecraft parameters and the environment, and would thus likely fit the intermittent or seasonal pattern. Quantitative analysis of this has been attempted, but is very complicated and beyond the scope of this paper. Nevertheless the possibility exists and it has not been conclusively disproved as a viable bypothesis.

\section{Boom Flexure}

The solar-induced bending of gravity-gradient booms has been known to cause anomalous attitude motion for some time. In general, the motion has been attributed to unexpected, undamped oscillations of torsionally weak, open section booms ${ }^{89,17}$. "Zippering" the gravity-gradient tape booms, as was done for Polar BEAR's boom, substantially

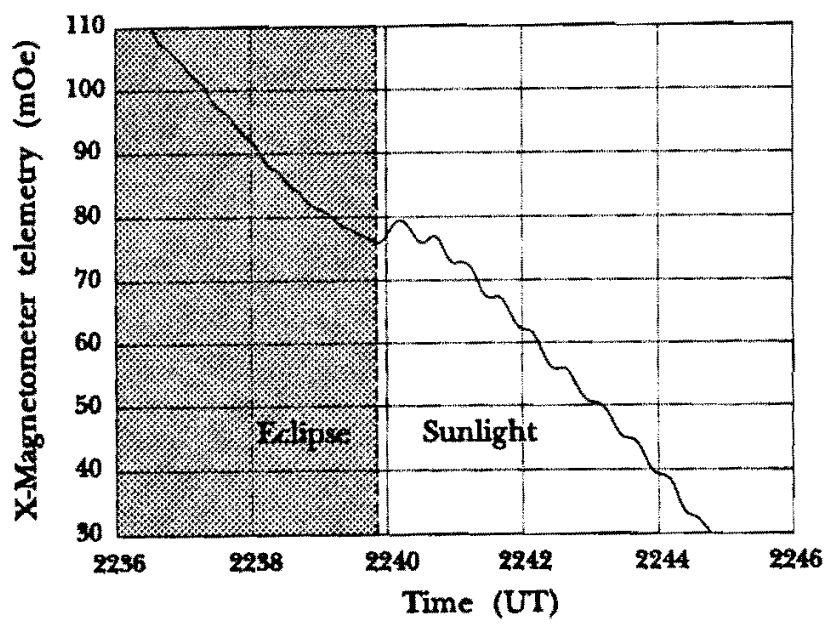

Figure 6 Evidence of Polar BEAR boom oscillation caused by thermal twang (day 44,1987 ).

increases torsional stiffness, and thermal flutter generally disappears. Thermal boom vibrations as a satellite enters and leaves the Earth's shadow still exist but do not cause spacecraft attitude instabilities. Polar BEAR boom oscillations are seen in the processed magnetometer telemetry shown in Fig. 6.

Investigations into the Polar BEAR anomaly have focussed on the thermal dynamics of the gravity-gradient boom. The Polar Bear boom is made of $51-\mu \mathrm{m}$-thick, silver-plated, beryllium copper formed into an interlocked tube $1.27-\mathrm{cm}$ in diameter. Solar radiation produces thermal gradients in the boom material, inducing deformation of the deployed boom element. The deformation is approximately $0.5-\mathrm{m}$ at the end of the $18.3-\mathrm{m}$ boom for normal solar incidence.

As the satellite's gravity-gradient boom deforms, the spacecraft's mass properties change. Associated with each new boom deformation state is a new spacecraft axis of minimum moment-of-inertia. Gravity-gradient torques continuously attempt to align the principal axis of minimum 
moment-of-inertia with the local vertical. Also, as the spacecraft attitude changes, the solar input on the boom changes, resulting in a different thermal equilibrium position. If the thermal boom deflection were acting in-phase with the attitude motion, it could potentially resonate with the attitude motion, increase the attitude errors, and with insufficient damping, lead to an instability. Instability of NRL's satellite 164 , which uses three interlocking tape booms, has been attributed to quasi-static thermal bending of the booms ${ }^{18}$. Simulations of quasi-static bending of the Polar BEAR boom did not predict its behavior, however.

\section{SIMUIATING POLAR BEAR'S ANOMALY}

Simulation of satellite attitude dynamics has played an important role in trying to understand the Polar BEAR anomaly. The Polar BEAR inversion appears to be caused by subtleties instead of some gross misbehavior. Analysis and modeling of most disturbances are unable to predict the Polar BEAR motion ${ }^{19}$. A non-linear simulation of Polar BEAR that models all of the major known disturbances and enables modeling of boom-bending effects has resulted in a possible explanation for the Polar BEAR motion.

\section{Boom Modeling}

The Polar BEAR interlocked, BI-STEM boom built by Astro Research Corporation, is formed from two thin, silverplated beryllium-copper alloy tapes. As they deploy, prestress in the tapes causes them to curl together forming a tubular boom with tabs and slots interlocking to form seams. Prestress also causes the seams to spiral along the length of the boom, enhancing straightness ${ }^{20}$.

The slots, tabs, and spiraling seam make detailed computation of boom thermal deflection difficult. When the boom is heated by the sun it assumes a shape that includes deflection both in the plane containing the longitudinal axis of the undeformed boom and the sun, and deflection out of that plane. Additionally, the boom may twist. Test results of short segments of interlocked booms show a variation in the amount of bending, both in-plane and out-of-plane, as the boom is rotated about its longitudinal axis relative to the heat source ${ }^{21}$.

Using test results of thermal boom bending, the Polar BEAR bending model was developed ${ }^{22}$. Defining "in-plane bending" as deformation of the boom away from the sun in the plane containing the spacecraft $z$-axis (nominal boom axis) and the sun, and "out-of-plane" bending as the deformation normal to that plane, we modeled thermal boom deformation to be:

$$
\begin{gathered}
\delta_{i}=\Delta \cdot \cos \theta \cdot\left[1-\sigma_{i} \cdot \cos \left(\phi-\phi_{i}\right)\right] \\
\delta_{0}=\Delta \cdot \cos \theta \cdot\left[-\sigma_{o} \cdot \sin \left(\phi-\phi_{0}\right)\right]
\end{gathered}
$$

where $\delta_{i}$ and $\delta_{o}$ are the amounts of in-plane and out-ofplane bending, respectively, $\Delta$ is the nominal static deflection for normal solar incidence, $\sigma_{i}$ and $\sigma_{o}$ are the percentage bending variations in the in-plane and out-of-plane directions, respectively. $\theta$ is the angle that the spacecraftto-sun line makes with the spacecraft $x-y$ plane (for $\theta=0^{\circ}$ the sun is normal to the undeformed boom). The sun azimuth angle in the $x-y$ plane (measured from the $y$-axis, positive about $z$ ) is given by $\phi_{i}$ and $\phi_{0}$ for the in-plane and out-of-plane bending phase angles, respectively. This bending model (which ignores boom twist) was used in the computer simulation. We are making the assumption that the boom's thermal time constant is much less than the fundamental mechanical bending period $(30-\mathrm{sec})$, so that the thermally bent position is an equilibrium position about which mechanical vibrations occur.

\section{Simulation Results}

The computer program, MULTIBOD ${ }^{23}$, was used to simulate the dynamics of the gravity-gradient stabilized spacecraft with a flexible boom. Figure 7 shows the relevant

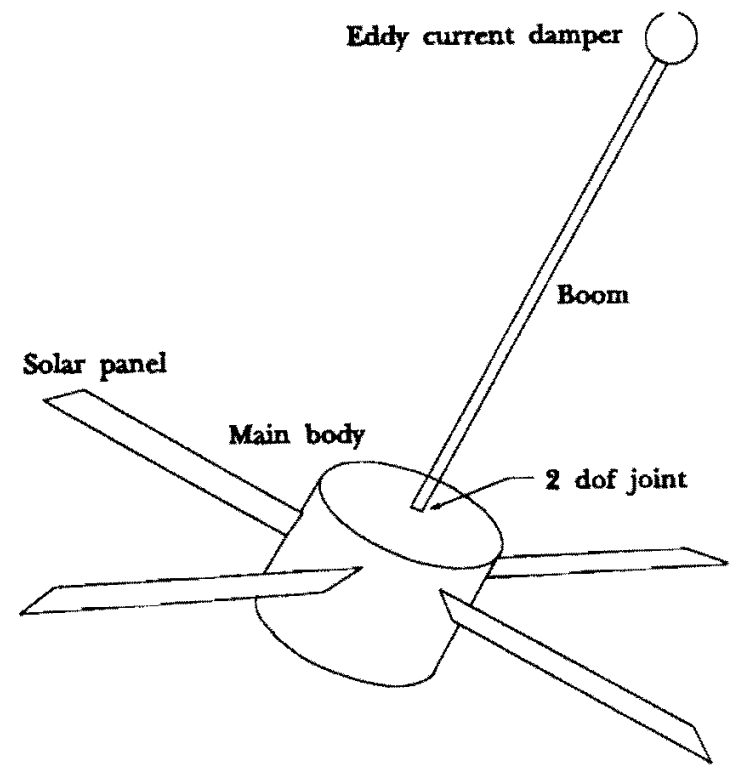

Figure 7 Polar BEAR simulation model.

features of the model. Two bodies are used, Polar BEAR's main body and the 18-m boom gravity-gradient boom/eddycurrent da mper combination. The constant speed momentum wheel is included in the main body. For aerodynamic and solar torques, the main body is modeled as 19 objects, including four flat plates for solar panels and several 
cylinders for antennas, while the boom is modeled as two objects (a long cylinder with a sphere at the end). The boom is attached to the main body through a two degree of freedom joint allowing both in-plane and out-of-plane bending, but not twist. Springs and dampers are specified for each hinge to give fundamental bending modes and structural damping for the Polar BEAR boom.

The thermal boom bending model described above, is incorporated into the simulation as a pair of thermal bias angles at the hinge about which the spring torques act. Spring torques are proportional to the deviation of the hinge angles from the bias angles. The bias angles are dynamically adjusted according to the angle between the spacecraft-Sun line and the direction along the boom's length.

Many simulation cases were run to investigate potential instabilities of Polar BEAR resulting from the dynamic thermal bending model. All cases were initialized with the same set of moderate attitude angles $\left(<10^{\circ}\right)$ and rates. Parameters that were varied included each of the boom bending model parameters and the right ascension of the sun relative to the orbital plane.

With no in-plane and out-of-plane bending variation ( $\sigma_{i}$ $=\sigma_{0}=0$ ), the simulations exhibit system stability for all sun angles. The zero bending variation cases model thermal bending as the in-plane thermal deformation of a uniform beam. This model is comparable to the quasi-static theory suggested by Goldman ${ }^{18}$ and was the approach taken in prelaunch simulations of Polar BEAR. The satellite described by Goldman had no momentum wheel, but instead used 3 gravity-gradient rods to achieve triaxial gravity-gradient stabilization.
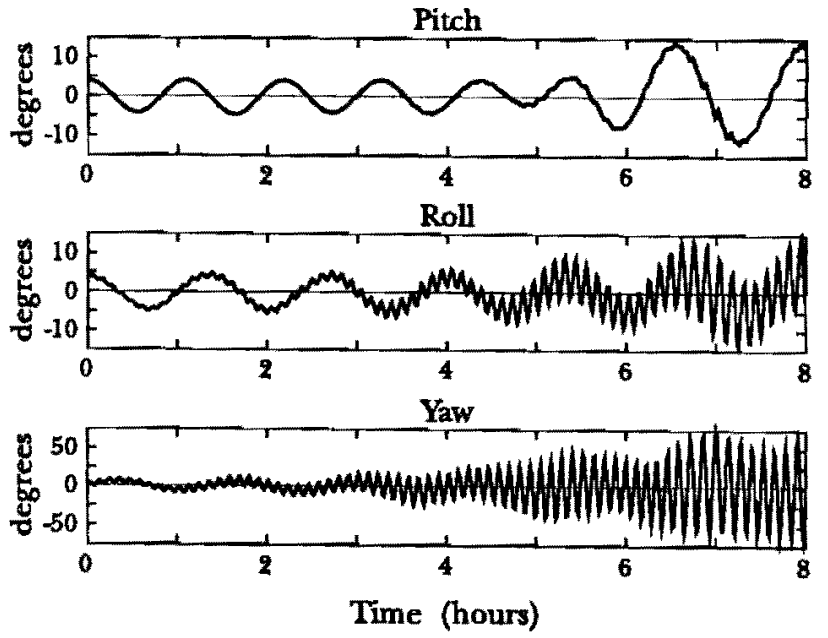

Figure 8 Simulated unstable motion.
The introduction of in-plane and out-of-plane bending variations to the simulation produced interesting results. For certain combinations of thermal bending phase angles $\left(\phi_{i}\right.$, $\phi_{o}$ ), the rigid-body fast roll/yaw mode became unstable for full-sun orbits (Figure 8). As expected, changing the phase angles by $180^{\circ}$ improved stability (Figure 9).
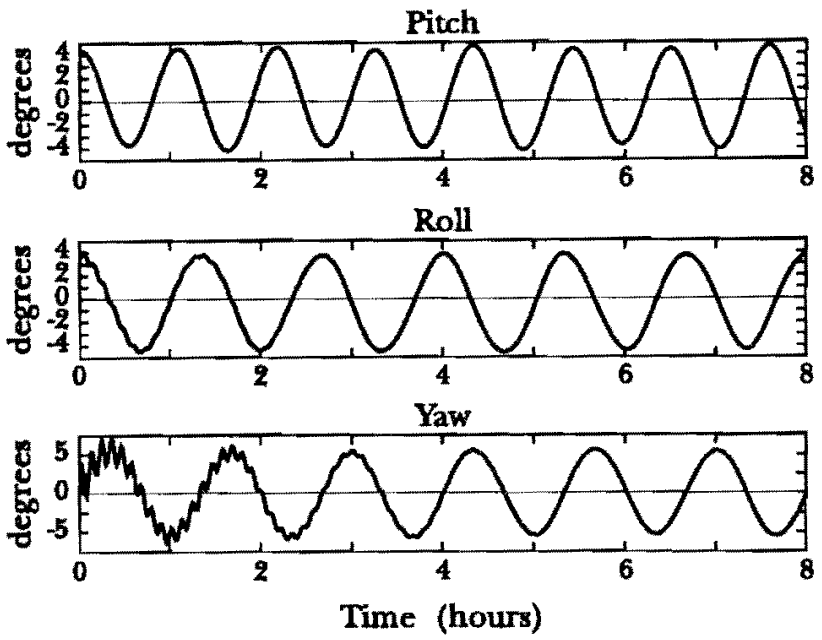

Figure 9 Simulated stable motion.

The apparent thermal instability suggested by the simulations produces roll/yaw divergence of the high frequency mode similar to that observed with Polar BEAR. It should be pointed out that the simulated roll/yaw divergence is much faster than the actual divergence ( 8 hours vs. 3-5 days); however, we believe that the bending parameters can be tweaked to produce behavior similar to that observed. In the interest of having manageable simulations we chose conservatively high values for the bending variation. The thermal bending model used is the only mechanism, thus far, that predicts the Polar BEAR motion.

Polar BEAR has only exhibited the apparent highfrequency roll/yaw instability during full-sun orbits. During eclipsed orbits thermal twang has been observed (and simulated) but the attitude has remained stable. The simulation, however, was able to produce a similar high frequency roll/yaw divergence for a few eclipsed orbit cases. This may be a further example of the subtleties associated with the flexible boom configuration.

\section{Inconsistent Full-Sun Performance}

While the simulation was able to produce motion like that of Polar BEAR it does not address why Polar BEAR inverts in some full-sun periods but not others. One possible explanation is that the boom is constantly changing 
orientation relative to the spacecraft and sun due to thermal twist. Its configuration entering each full-sun period is dependent upon its activity prior to that period. Although torsionally rigid, the interlocked BI-STEM has a large torsional backlash zone (or dead zone) in which the boom acts like a torsionally-weak overlapped boom ${ }^{24}$. Consequently, it is entirely plausible that the thermal phase angle could vary by $180^{\circ}$ between full-sun periods. When the boom twists due to the spacecraft dynamics or thermal deformation, it may remain within its backlash zone and therefore not return to the previous orientation when the stress is removed.

\section{CONCLUSIONS}

There are many potential causes for the Polar BEAR anomaly; it is still not well understood. Quite likely, there are many different interactions working together to produce the undesirable attitude motion; if taken individually these interactions may be unable to produce an instability. Simulations suggest that variations in thermal bending of the gravity-gradient boom can produce instabilities like Polar BEAR's, but the results are in conclusive.

Future missions planning to use an attitude control design like Polar BEAR's (BI-STEM gravity-gradient boom, momentum wheel, and eddy current damper) should be aware of the difficulties that have been experienced with the configuration. All of the spacecraft with these problems have recovered, but seasonal or sporadic upsets should not be a surprise. Preparations for anomaly recovery should be part of the pre-launch mission planning.

Alternative configurations exist but are, not surprisingly, generally more expensive. The same type of gravitygradient configuration works quite well for spacecraft with stiff booms that experience little, if any, thermal deformation. The Navy's NOVA series of satellites use the truss-structured Astromast and achieve on the order of 5-10 pointing performance. GEOSAT and its predecessor GEOSIII both used very stiff scissors booms with $45-\mathrm{kg}$ end masses to achieve $1-2^{\circ}$ nadir-pointing. Finally, active pitch control with momentum-bias for roll/yaw stabilization is becoming affordable as small satellites gain in popularity.

\section{ACKNOWLEDGEMENTS}

We would like to thank W. L. Ebert and H. L. Fisher for their efforts and ideas during the anomaly study. The spacecraft charging hypothesis is by P. J. Melvin. We also appreciate the in-orbit attitude data for trend analysis, which was provided anonymously.

\section{REFERENCES}

${ }^{1}$ Roberson, R. E., "Gravitational Torque on a Satellite Vehicle," J. Franklin Inst. 265, 13-22 (1958).

${ }^{2}$ Pisacane, V. L., Three-Axis Stabilization of a Dumbbell Satellite by a Small Constant-Speed Rotor, JHU/APL TG 855 (Oct 1966).

${ }^{3}$ Peterson, M. R., and Grant, D. G., "The Polar BEAR Spacecraft," Johns Hopkins APL Tech. Dig. Vol. 8, no. 3, 295-302 (1987).

${ }^{4}$ Hunt, J. W., and Williams, C. E., "Anomalous Attitude Motion of the Polar BEAR Satellite," Johns Hopkins APL Tech. Dig. Vol. 8, no. 3, 324-328 (1987).

${ }^{5}$ Williams, C. E., "Polar BEAR Attitude Performance During September 1987," JHU/APL S3G-87-264, 29 Sep. 1987.

'Potocki, K. A., "The HILAT Spacecraft," Johns Hopkins APL Tech. Dig. Vol. S, no. 2, 104-108 (1984).

${ }^{7}$ Eisner, A. and Heyler, G. A., "HILAT Attitude Performance," JHU/APL S1A-117-84, 8 Oct. 1984.

${ }^{8}$ Frisch, H. P., "Thermally Induced Vibrations of Long Thin-Walled Cylinders of Open Section, " J. Spacecraft and Rockets, vol. 7, no. 8, 897-905 (Aug 1970).

${ }^{9}$ Hughes, P. C., Spacecraft Attitude Dynamics, John Wiley \& Sons, New York (1986).

${ }^{10 n}$ Flight Analysis of Naval Research Laboratory SemiPassive Stabilization Subsystem," General Electric Doc. No. 70SD5305, 17 July 1970.

${ }^{11}$ Johns Hopkins APL Tech. Dig. Vol. 8, no. 2 (1987).

${ }^{12}$ Kilgus, C. C., "GEOSAT Coverage of the Ocean," JHU/APL SDO-8497, 3 June 1987.

${ }^{19}$ Eisner, A. and Yionoulis, S. M., "NOVA-1 The Drag-Free Navigation Satellite," National Aerospace Symposium of the Institute of Navigation, NASA AMES Research Center, Moffett Field, CA, 23-25 March 1982.

${ }^{14}$ Spacecraft Attitude Determination and Control, J. R. Wertz, ed., D. Reidel Publishing Co., Dordrecht, Holland (1978). 
${ }^{15}$ Pisacane, V. L., "NOVA Attitude Motion in the Presence of Torque Biases, "JHU/APL SD0-6024, June 16, 1981.

${ }^{16}$ Garber, T. B., "Influence of Constant Disturbing Torques on the Motion of Gravity-Gradient Stabilized Satellites," AIAA Journal, v. 1, no. 4 (April 1963).

${ }^{17}$ Graham, J. D., "Solar Induced Bending Vibrations of a Flexible Member," AIAA J. 8, 2031-2036 (1970).

${ }^{18}$ Goldman, R. L., "Influence of Thermal Distortion on Gravity Gradient Stabilization," J. Spacecraft, vol. 12, no. 7, 406-413 (1975).

${ }^{19}$ Lewis, A. and Streland, A., "Instability of Gravity Gradient Spacecraft in Full Sun Orbit: Flight Experience from the Polar Bear Mission," Guidance and Control 1989, Advances in the Astronautical Sciences, Vol. 68, 505-531 (1989).
${ }^{20}$ Astro Research Corporation, "Interlocked BI-STEM Boom 0.5-Inch-Diameter," ARC-B-008 (April 1979).

${ }^{21}$ Staugaitis, C. L., and Predmore, R. E., "Thermal Static Bending of Deployable Interlocked Booms," NASA TN D7243 (March, 1973).

${ }^{2}$ Ebert, W. L., "Simulation of Bi-Stem Boom Dynamic Bending," JHU/APL S3G-89-057, 15 March 1989.

${ }^{23}$ Hunt, J. W. and Fisher, H. L., "Simulating the Attitude Dynamics of a Multibody Spacecraft," Proc. 1990 Summer Computer Simulation Conference, Calgary, Alberta, Canada, (July, 1990).

${ }^{24}$ Laskin, R. A., "Thermoelastic Limit Cycling of Zippered Cross Section Booms," AlAA Paper No. 84-1065CP (1984). 